\title{
PEG-Assisted DNA Solubilization in Organic Solvents for Preparing Cytosol Specifically Degradable PEG/DNA Nanogels
}

\author{
Hyejung Mok, Tae Gwan Park* \\ ${ }^{I}$ Department of Biological Sciences, Korea Advanced Institute of Science and \\ Technology, Daejeon, South Korea
}

\section{Supplementary materials: Experimental details}

1. DNA solubilization in organic solvents

Poly(ethylene glycol) (PEG, Mw 3,350) was purchased from Sigma (St. Louis, MO).

Dialysis membrane (MW cut-off 50,000) was purchased from Spectrum (Houston, TX).

Salmon sperm DNA from Stratagene (La Jolla, CA) was used for DNA solubilization test after dialysis for desalting. Plasmid DNA (pEGFP-C1) was extracted from transformed Escherichia coli by a standard alkaline lysis technique and purified by a DNA-purification column which was purchased from QIAGEN (Valencia, CA). The purity and concentration of plasmid DNA were determined by measuring absorbance 
ratio at 260/280 $\mathrm{nm}$ using a spectrophotometer (UV-1601, Shimadzu, Japan). DNA was ethanol-precipitated and resuspended in deionized water. The residual ion concentration was low $(<<10 \mathrm{mM})$. Salt-free salmon sperm DNA or plasmid DNA $(1 \mathrm{mg})$ was mixed with PEG with varying weight ratios in deionized water at room temperature and freezedried. A dry mixture of DNA and PEG was added in various organic solvents (ethanol, methanol, DMSO, acetone, hexane, ethyl acetate, methylene chloride, and THF). The final concentration of DNA in solvent was $0.4 \mathrm{mg} / \mathrm{ml}$. After solubilzing DNA in organic solvent, transmittance was determined by measuring transmittance (\%) at $400 \mathrm{~nm}$ using a spectrophotometer (UV-1601, Shimadzu). Pure DMSO solution was used as a control (100\% transmittance). The DNA solubility in organic solvent was determined by measuring the amount of DNA in the supernatant (measuring absorbance at $260 \mathrm{~nm}$ ) after centrifuging at $14000 \mathrm{rpm}$ and dialyzing the supernatant in deionized water. The solubilized plasmid DNA in DMSO or methanol $(0.1 \mathrm{mg} / \mathrm{ml})$ was subjected to take circular dichroism (CD) spectra. The CD (JASO J710, Japan) spectra were obtained at $25{ }^{\circ} \mathrm{C}$.

\section{Preparation of PEG/DNA nanogels}

Six-arm branched and thiol funcionalized PEG derivative (Mw 20,000) was 
purchased from Sunbio (Anyang City, Korea). Six-arm branched PEG-SH (7.5 mg) and plasmid DNA $(0.5 \mathrm{mg})$ were co-dissolved in $2 \mathrm{ml}$ of DMSO, to which $10 \mathrm{mg}$ of dithiobis-maleimidoethane (DTME) in $1 \mathrm{ml}$ of DMSO was added to the PEG/DNA complex with stirring. After overnight reaction, the solution was centrifuged at $14000 \mathrm{rpm}$ for $1 \mathrm{~h}$ to remove free DTME and uncross-linked PEG and DNA. PEG nanogels collected in the pellet were resuspended with deionized-water.

\section{AFM (Atomic Force Microscopy)}

Morphological characters of DNA in deionized water, PEG/DNA complex in DMSO, and PEG nanogels in deionized water were observed by AFM. Samples were loaded on a freshly cleaved-mica surface and dried in a vacuum oven at room temperature. AFM measurements were done by using a $50 \times 50$ um scanner of PSIA XE-100 AFM system (Santa Clara, CA, USA) in a non-contact mode. The scanned image was collected from a $3 \times 3$ or $5 \times 5 \mu \mathrm{m}$ area.

\section{Characterization of PEG/DNA nanogels}

Particle size and surface charge values were measured by using a dynamic light scattering instrument (Zeta plus, Brookhaven, New York) equipped with a He-Ne laser 
at a wavelength of $632.2 \mathrm{~nm}$. Nanogels in $3 \mathrm{ml}$ of deionized water were analyzed at room temperature. PEG nanogels were prepared at varying DTME crosslinker/PEG-SH molar ratios from 1 to 15 . To determine the loading amount of plasmid DNA, dithiothreitol (DTT) $(100 \mathrm{mM})$ was added to dissolve the PEG nanogels. The amount of six-arm branched PEG-SH in the nanogels was measured by Ellman's assay and the DNA amount was determined by Picogreen assay (Molecular Probe, Eugene, OR). The amount of free -SH in the PEG nanogels was also determined by an Ellman's assay without DTT treatment.

\section{Degradation of PEG/DNA nanogels}

The PEG nanogels cross-linked by disulfide-bonds were analyzed by agarose gel electrophoresis. DTT (100 mM) treated or no-treated PEG nanogels (16 $\mu \mathrm{g}$ PEG-SH, $0.9 \mu \mathrm{g}$ DNA) were loaded on $1 \%$ agarose gel. Electrophoresis was carried out with a current of $100 \mathrm{~V}$ for $20 \mathrm{~min}$ in TAE buffer solution (40 mM Tris-HCl, $1 \%$ (v/v) acetic acid, and $1 \mathrm{mM}$ EDTA). DNA encapsulated within the nanogels was visualized by staining with ethidium bromide. For mimicking intracellular condition, gluthathione (GSH) was used as a reducing agent. GSH was added to the PBS solution ( $\mathrm{pH} 7.4$ ) containing PEG/DNA nanogels at concentrations of $0,0.1$, and $5 \mathrm{mM}$. At pre- 
determined time intervals, the supernatant was collected by centrifuging at $14000 \mathrm{rpm}$ for $30 \mathrm{~min}$ and the amount of the released DNA in the incubation medium was determined by Picogreen assay.

6. Intracellular uptake and gene delivery experiment using PEG/DNA nanogels

HeLa cells or HEK293 cells were seeded at a density of 1 X $10^{5}$ cells/well in a 4-well chamber-slide and kept overnight. PEG nanogels encapsulating pEGFP-C1 plasmid DNA were treated to HeLa cells in serum-free media for $1 \mathrm{hr}$ and washed with PBS. After further incubation in $10 \%$ serum medium for $0 \mathrm{hr}$ or $4 \mathrm{hr}$, the cells were washed with cold PBS three times and fixed in $3.7 \%$ formaldehyde solution for 30 min on ice. The slides were covered with a VECTASHIELD® Mounting Medium with DAPI (Vector Laboratories, Inc., Burlingame, CA). Plasmid DNA within PEG nanogels were stained with DAPI (4', 6-diamidino-2-Phenylindole) dye and imaged with a confocal laser scanning microscope (LSM 510, CARL-ZEISS, USA) .

After the plasmid DNA encapsulated within PEG nanogels was delivered, the expression of GFP plasmid was examined in HEK293 cell line. For enhancement of endosome escape, KALA peptide (WEAKLAKALAKALAKHLAKALAKALKACEA, Peptron, Korea) was coated onto the surface of PEG/DNA nanogel. After transfection 
with PEG gel and PEG gel+KALA peptide, cells were incubated for 2 days and imaged with a confocal microscope.

\section{Supplementary data}

\section{Additional figures}

\section{Figure Captions}

Figure S1. (a) Photograph and (b) transmittance of the DMSO solution in which DNA was dissolved at various PEG/DNA weight ratios. Final concentration of salmon sperm DNA in organic solvent was $0.4 \mathrm{mg} / \mathrm{ml}$.

Figure S2. Dissolved DNA amount in DMSO at various PEG/DNA weight ratios.

Figure S3. Atomic force microscopy image of PEG/DNA nanocomplex (weight ratio PEG/DNA=1) in DMSO.

Figure S4. Atomic force microscopy image of PEG/DNA nanogel in water.

Figure S5. GFP expression of plasmid DNA using PEG nanogels in HEK 293 cells. 
a.

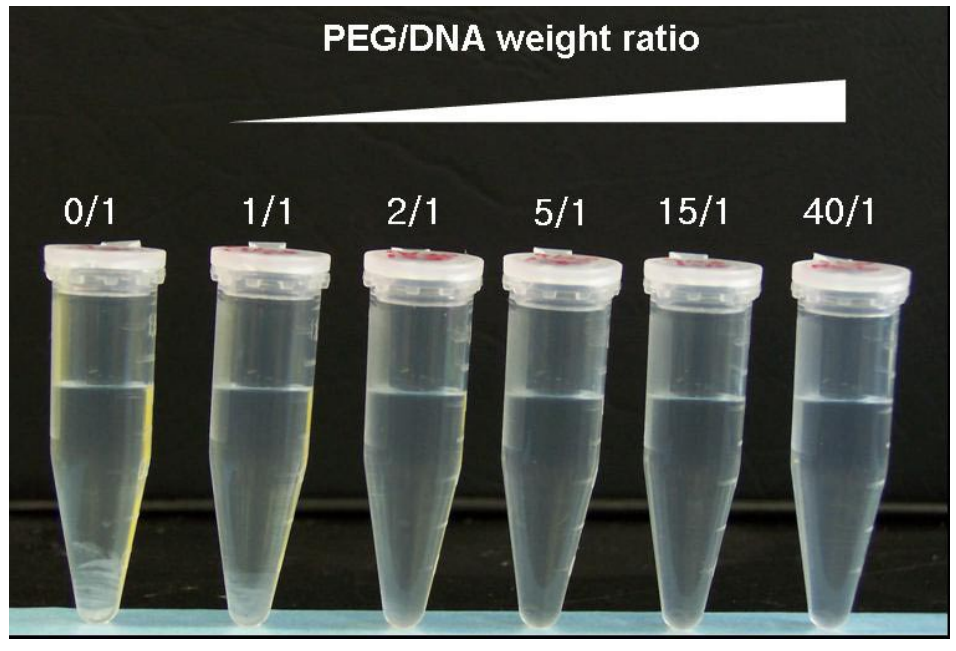

b.

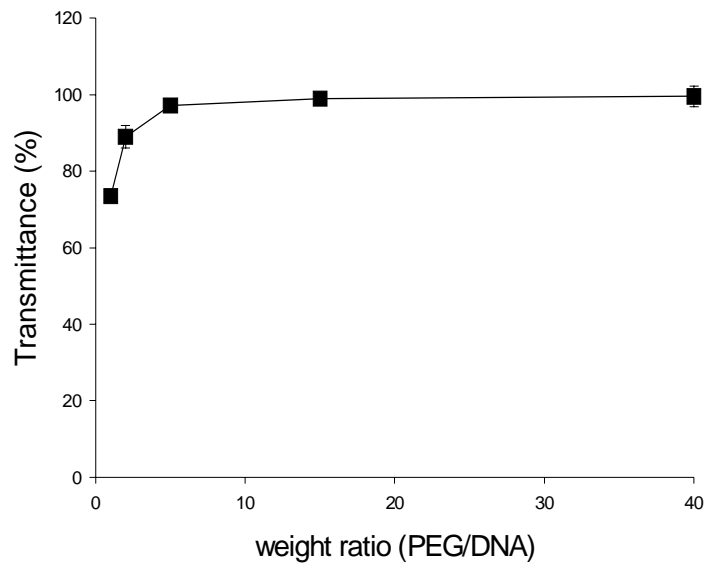

Figure S1 


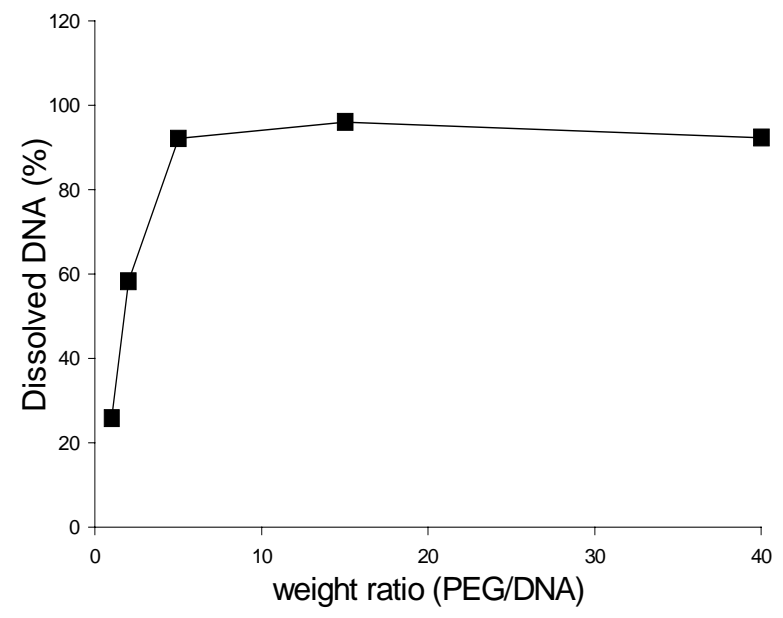

Figure S2 


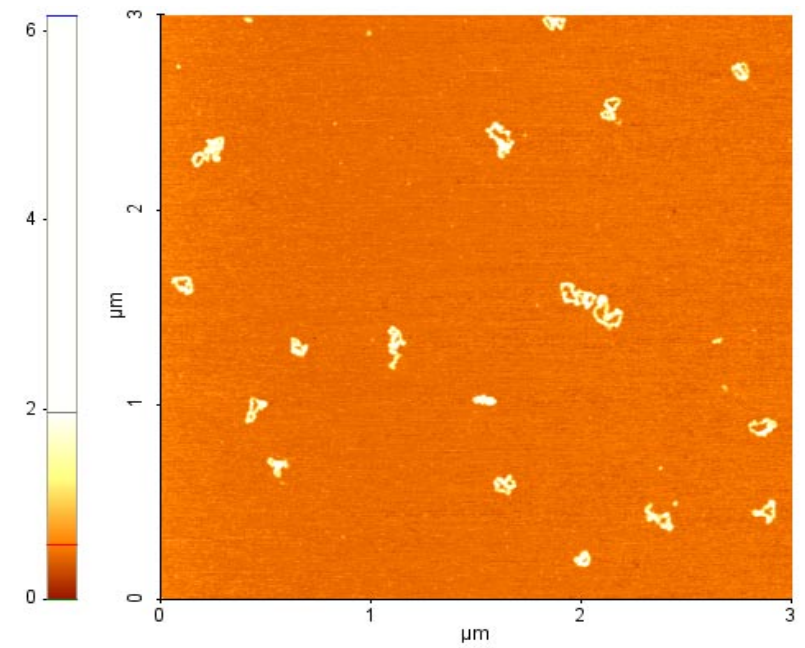

Figure S3 


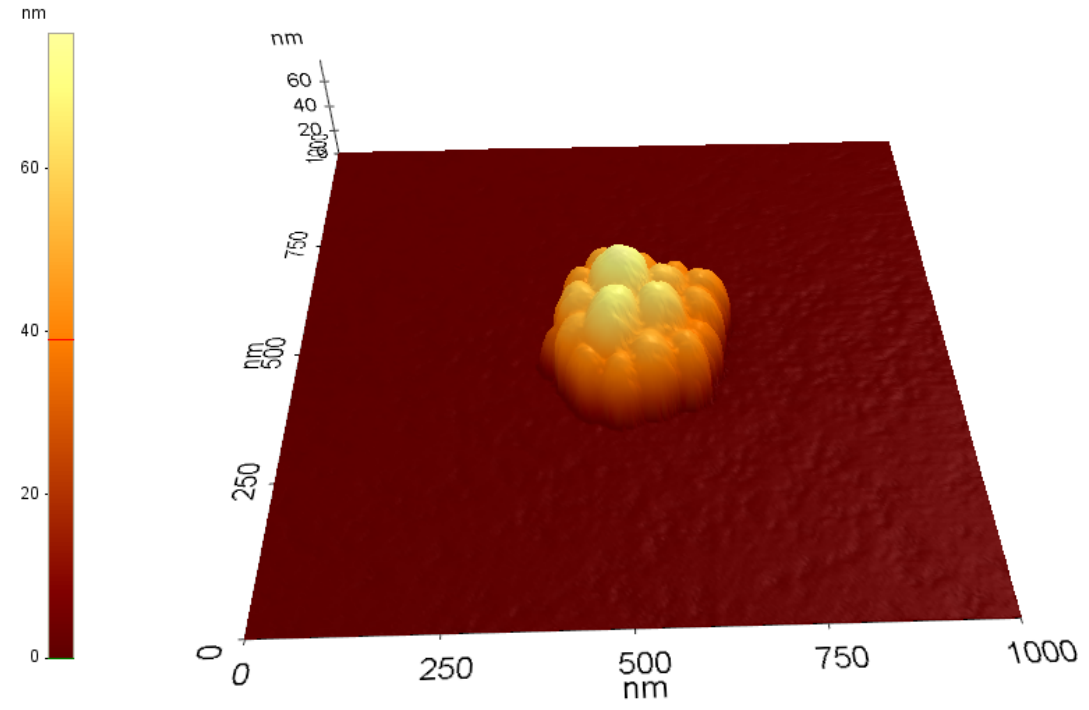

Figure S4 

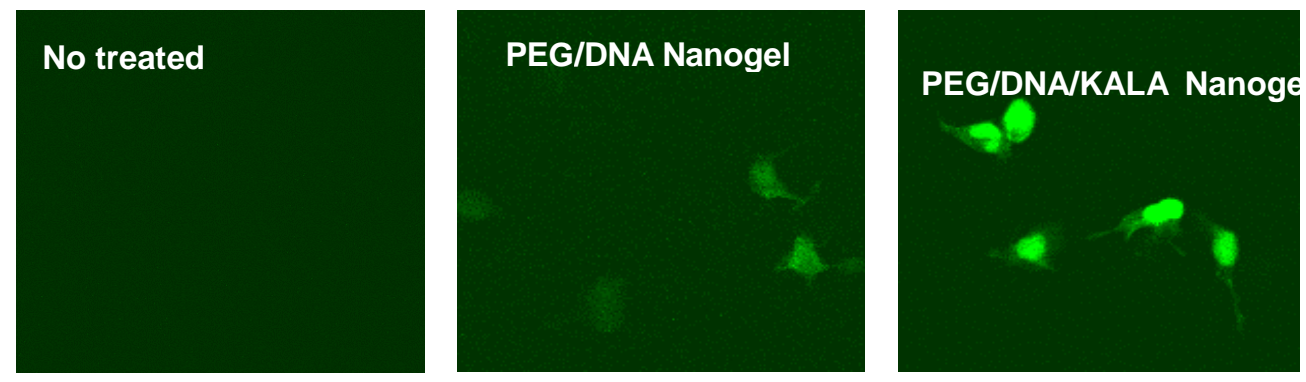

Figure S5 